Acta Crystallographica Section E

Structure Reports

Online

ISSN 1600-5368

\section{$\mu$-Propane-1,3-dithiolato- $\kappa^{4} S, S^{\prime}: S, S^{\prime}$-bis- [dicarbonyl(triphenylphosphane- $\kappa P$ )- $\operatorname{iron}(\mathrm{II})](\mathrm{Fe}-\mathrm{Fe})$}

\section{Bang-Shao Yin,* Tian-Bao Li and Ming-Sheng Yang}

College of Chemistry and Chemical Engineering, Hunan Normal University, Hunan 410081, People's Republic of China

Correspondence e-mail: yinbangshao@yahoo.cn

Received 30 September 2011; accepted 3 October 2011

Key indicators: single-crystal X-ray study; $T=113 \mathrm{~K}$; mean $\sigma(\mathrm{C}-\mathrm{C})=0.011 \AA$; $R$ factor $=0.075 ; w R$ factor $=0.146 ;$ data-to-parameter ratio $=14.0$.

The title compound, $\left[\mathrm{Fe}_{2}\left(\mathrm{C}_{3} \mathrm{H}_{6} \mathrm{~S}_{2}\right)\left(\mathrm{C}_{18} \mathrm{H}_{15} \mathrm{P}\right)_{2}(\mathrm{CO})_{4}\right]$, which might serve as an active-site model of [FeFe]-hydrogenase, contains two fused $\mathrm{Fe} / \mathrm{S} / \mathrm{C} / \mathrm{C} / \mathrm{C} / \mathrm{S}$ six-membered rings, one of which has a chair conformation and the other a boat conformation. Each Fe atom is coordinated by two carbonyl ligands, a triphenylphosphane ligand and a bis-bidentate dithiolate ligand, and also forms an $\mathrm{Fe}-\mathrm{Fe}$ bond [2.5167 (16) §]. Together, the six bonded atoms form a very distorted octahedral arrangement.

\section{Related literature}

For details of the synthesis, see: Li et al. (2005). For more details about [FeFe]-hydrogenase model complexes, see: Song et al. (2005); Liu \& Xiao (2011); Liu \& Yin (2010, 2011); Liu et al. (2011).

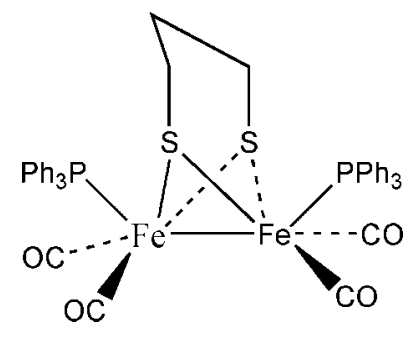

\section{Experimental}

Crystal data

$\left[\mathrm{Fe}_{2}\left(\mathrm{C}_{3} \mathrm{H}_{6} \mathrm{~S}_{2}\right)\left(\mathrm{C}_{18} \mathrm{H}_{15} \mathrm{P}\right)_{2}(\mathrm{CO})_{4}\right] \quad M_{r}=854.48$

$$
\begin{aligned}
& \text { Triclinic, } P \overline{1} \\
& a=9.139(5) \AA \\
& b=13.480(5) \AA \\
& c=16.786(10) \AA \\
& \alpha=77.773(19)^{\circ} \\
& \beta=89.50(2)^{\circ} \\
& \gamma=71.187(18)^{\circ}
\end{aligned}
$$

\section{Data collection \\ Rigaku Saturn724 CCD diffractometer \\ Absorption correction: multi-scan (CrystalClear; Rigaku/MSC, 2005) \\ $T_{\min }=0.943, T_{\max }=0.961$}

Refinement

$R\left[F^{2}>2 \sigma\left(F^{2}\right)\right]=0.075$

$w R\left(F^{2}\right)=0.146$

$S=0.97$

6709 reflections

Table 1

Selected bond lengths $(\AA)$.

\begin{tabular}{llll}
\hline $\mathrm{Fe} 1-\mathrm{C} 2$ & $1.719(8)$ & $\mathrm{Fe} 2-\mathrm{C} 4$ & $1.720(8)$ \\
$\mathrm{Fe} 1-\mathrm{C} 1$ & $1.773(8)$ & $\mathrm{Fe} 2-\mathrm{C} 3$ & $1.750(8)$ \\
$\mathrm{Fe} 1-\mathrm{P} 1$ & $2.237(2)$ & $\mathrm{Fe} 2-\mathrm{P} 2$ & $2.230(2)$ \\
$\mathrm{Fe} 1-\mathrm{S} 2$ & $2.254(2)$ & $\mathrm{Fe} 2-\mathrm{S} 1$ & $2.276(2)$ \\
$\mathrm{Fe} 1-\mathrm{S} 1$ & $2.285(2)$ & $\mathrm{Fe} 2-\mathrm{S} 2$ & $2.287(2)$ \\
\hline
\end{tabular}

Data collection: CrystalClear (Rigaku/MSC, 2005); cell refinement: CrystalClear; data reduction: CrystalClear; program(s) used to solve structure: SHELXS97 (Sheldrick, 2008); program(s) used to refine structure: SHELXL97 (Sheldrick, 2008); molecular graphics: XP in SHELXTL (Sheldrick, 2008); software used to prepare material for publication: CrystalStructure (Rigaku/MSC, 2005).

This work was supported by the start-up foundation of Hunan Normal University.

Supplementary data and figures for this paper are available from the IUCr electronic archives (Reference: HB6432).

\section{References}

Li, P., Wang, M., He, C., Li, G., Liu, X., Chen, C., Akermark, B. \& Sun, L. (2005). Eur. J. Inorg. Chem. pp. 2506-2513.

Liu, X. F. \& Xiao, X. W. (2011). J. Organomet. Chem. 696, 2767-2771.

Liu, X. F., Xiao, X. W. \& Shen, L. J. (2011). J. Coord. Chem. 64, 1023-1031.

Liu, X. F. \& Yin, B. S. (2010). J. Coord. Chem. 63, 4061-4067.

Liu, X. F. \& Yin, B. S. (2011). Z. Anorg. Allg. Chem. 637, 377-379.

Rigaku/MSC (2005). CrystalClear and CrystalStructure. Rigaku/MSC Inc., The Woodlands, Texas, USA.

Sheldrick, G. M. (2008). Acta Cryst. A64, 112-122.

Song, L. C., Yang, Z. Y., Bian, H. Z., Liu, Y., Wang, H. T., Liu, X. F. \& Hu, Q. M. (2005). Organometallics, 24, 6126-6135. 


\section{supporting information}

Acta Cryst. (2011). E67, m1502 [doi:10.1107/S1600536811040621]

\section{$\mu$-Propane-1,3-dithiolato- $\kappa^{4} S, S^{\prime}: S, S^{\prime}$-bis[dicarbonyl(triphenylphosphane- $\kappa P) \operatorname{iron}(\mathrm{II})](\mathrm{Fe}-\mathrm{Fe})$}

\section{Bang-Shao Yin, Tian-Bao Li and Ming-Sheng Yang}

\section{S1. Comment}

[FeFe]-hydrogenases are a class of natural enzymes that can catalyze the production and consumption of hydrogen gas in several microorganisms (Song et al., 2005); Liu \& Yin, 2010, 2011); Liu \& Xiao, 2011; Liu et al., 2011). In continuated our work, a $[\mathrm{FeFe}]-$ hydrogenases model complex had been synthesized. The strucuture was confirmed by X-ray crstallography.

Single-crystal X-ray diffraction analysis reveals that the title complex crystallizes in the triclinic space group P-1. As shown in Fig. 1, the title complex contains four carbonyls and two $\mathrm{PPh}_{3}$ ligands. The diiron propanedithiolate consists of two fused six-membered rings, in which one ring has a chair conformation and the other ring has a boat conformation. The $\mathrm{PPh}_{3}$ ligands reside in an axial position of the square-pyramidal geometry of the Fe atoms. As shown in Fig. 2, the crystal structure is stabilized by van der Waals interactions.

\section{S2. Experimental}

The title complex was prepared according to the literature procedures (Li et al , 2005)). Crystals were grown from slow evaporation of dichloromethane and hexane solution at room temperature.

\section{S3. Refinement}

All the $\mathrm{H}$ atoms were positioned geometrically $(\mathrm{C}-\mathrm{H}=0.93-0.97 \AA)$ and refined as riding with $U_{\text {iso }}(\mathrm{H})=1.2 U_{\text {eq }}(\mathrm{C})$ or $1.5 U_{\mathrm{eq}}($ methyl C). 


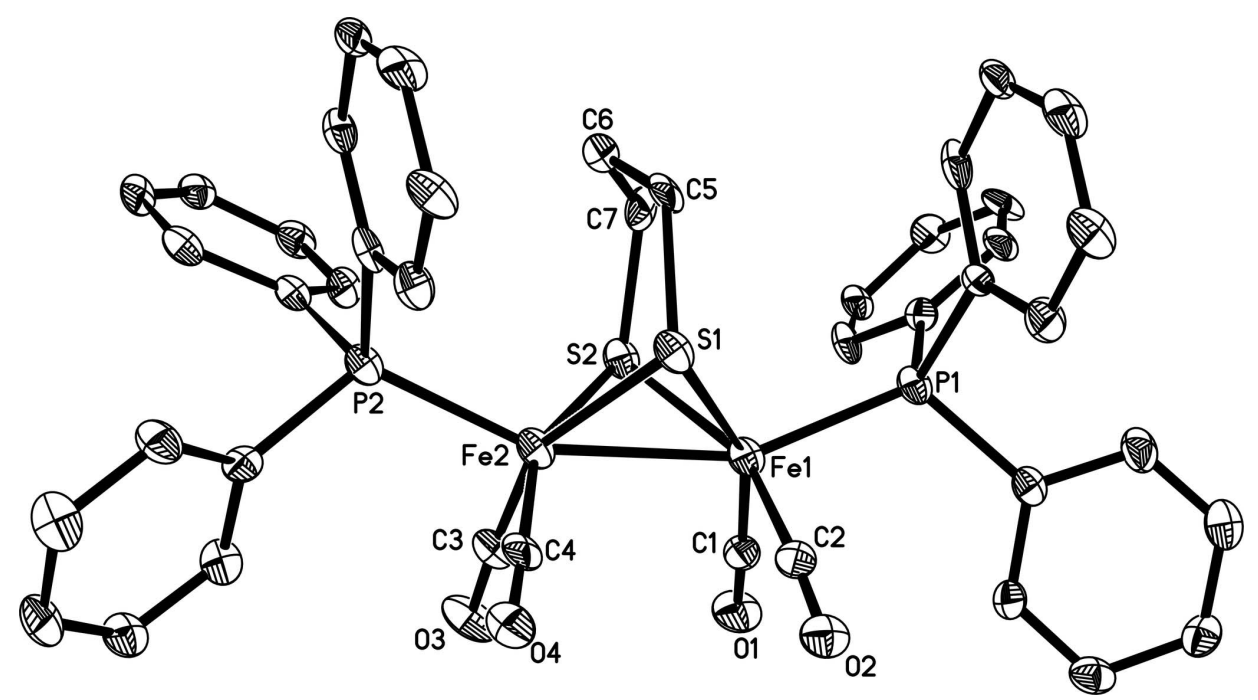

Figure 1

The molecular structure of (I). Displacement ellipsoids are drawn at the $30 \%$ probability level and $\mathrm{H}$ atoms are shown as small spheres of arbitrary radii.

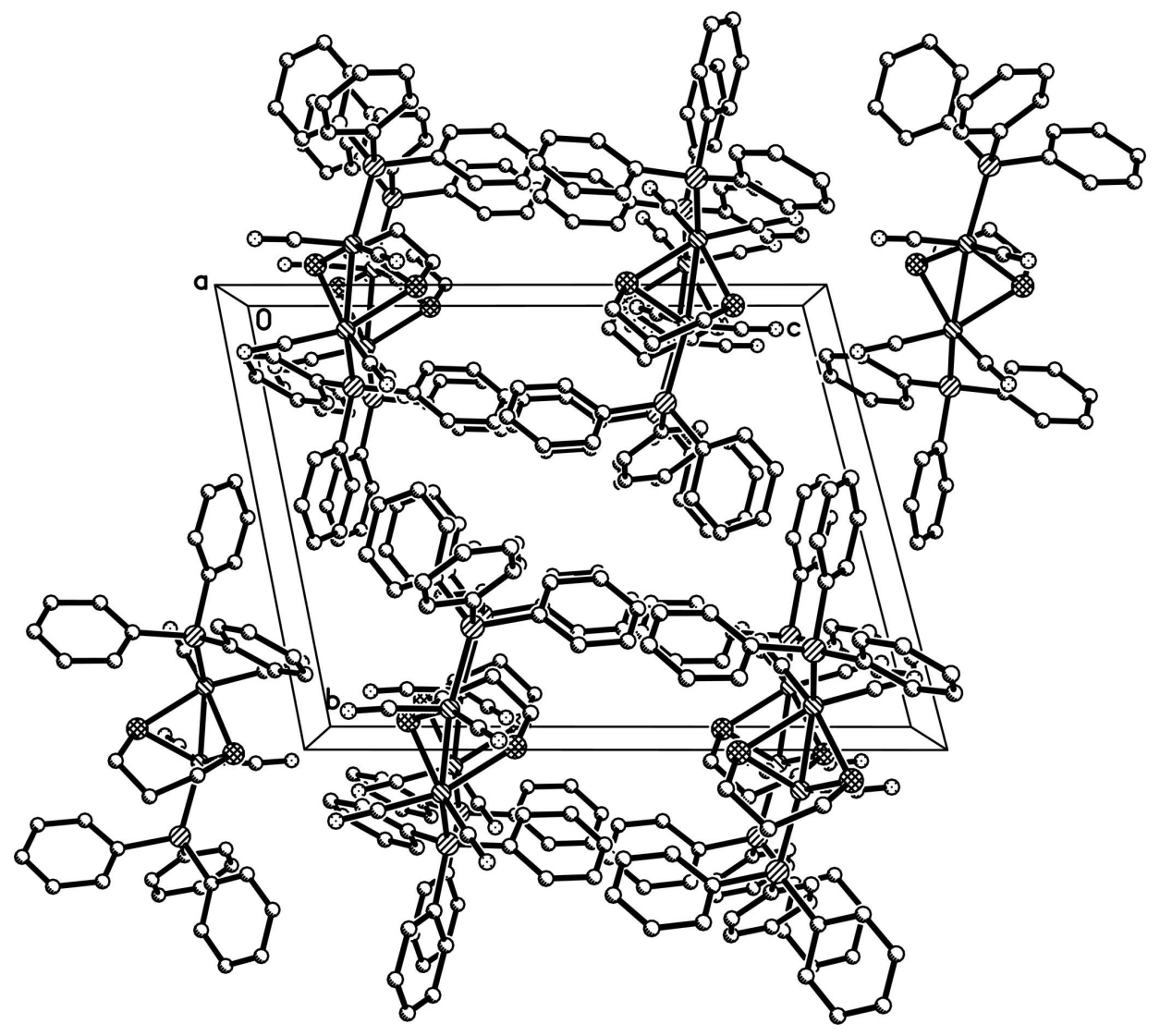

Figure 2

The crystal packing for (I). 
$\mu$-Propane-1,3-dithiolato- $\kappa^{4} S, S^{\prime}: S, S^{\prime}$ - bis[dicarbonyl(triphenylphosphane- $\left.\kappa P\right)$ iron(II)] $(\mathrm{Fe}-\mathrm{Fe})$

Crystal data

$\left[\mathrm{Fe}_{2}\left(\mathrm{C}_{3} \mathrm{H}_{6} \mathrm{~S}_{2}\right)\left(\mathrm{C}_{18} \mathrm{H}_{15} \mathrm{P}\right)_{2}(\mathrm{CO})_{4}\right]$

$M_{r}=854.48$

Triclinic, $P \overline{1}$

$a=9.139(5) \AA$

$b=13.480(5) \AA$

$c=16.786(10) \AA$

$\alpha=77.773(19)^{\circ}$

$\beta=89.50(2)^{\circ}$

$\gamma=71.187(18)^{\circ}$

$V=1909.1(17) \AA^{3}$

\section{Data collection}

Rigaku Saturn724 CCD

diffractometer

Radiation source: rotating anode

Multilayer monochromator

Detector resolution: 14.22 pixels $\mathrm{mm}^{-1}$

$\omega$ and $\varphi$ scans

Absorption correction: multi-scan

(CrystalClear; Rigaku/MSC, 2005)

$T_{\min }=0.943, T_{\max }=0.961$

\section{Refinement}

Refinement on $F^{2}$

Least-squares matrix: full

$R\left[F^{2}>2 \sigma\left(F^{2}\right)\right]=0.075$

$w R\left(F^{2}\right)=0.146$

$S=0.97$

6709 reflections

478 parameters

0 restraints

Primary atom site location: structure-invariant

direct methods
$Z=2$

$F(000)=880$

$D_{\mathrm{x}}=1.486 \mathrm{Mg} \mathrm{m}^{-3}$

Mo $K \alpha$ radiation, $\lambda=0.71073 \AA$

Cell parameters from 5985 reflections

$\theta=1.6-26.1^{\circ}$

$\mu=1.00 \mathrm{~mm}^{-1}$

$T=113 \mathrm{~K}$

Prism, colorless

$0.06 \times 0.04 \times 0.04 \mathrm{~mm}$

15996 measured reflections

6709 independent reflections

2718 reflections with $I>2 \sigma(I)$

$R_{\text {int }}=0.124$

$\theta_{\max }=25.0^{\circ}, \theta_{\min }=1.6^{\circ}$

$h=-10 \rightarrow 10$

$k=-16 \rightarrow 12$

$l=-19 \rightarrow 19$

Secondary atom site location: difference Fourier map

Hydrogen site location: inferred from neighbouring sites

$\mathrm{H}$-atom parameters constrained

$w=1 /\left[\sigma^{2}\left(F_{\mathrm{o}}{ }^{2}\right)+(0.0041 P)^{2}\right]$

where $P=\left(F_{\mathrm{o}}^{2}+2 F_{\mathrm{c}}^{2}\right) / 3$

$(\Delta / \sigma)_{\max }=0.001$

$\Delta \rho_{\max }=1.35 \mathrm{e} \AA^{-3}$

$\Delta \rho_{\min }=-0.66$ e $\AA^{-3}$

Special details

Geometry. All e.s.d.'s (except the e.s.d. in the dihedral angle between two 1.s. planes) are estimated using the full covariance matrix. The cell e.s.d.'s are taken into account individually in the estimation of e.s.d.'s in distances, angles and torsion angles; correlations between e.s.d.'s in cell parameters are only used when they are defined by crystal symmetry. An approximate (isotropic) treatment of cell e.s.d.'s is used for estimating e.s.d.'s involving 1.s. planes.

Refinement. Refinement of $F^{2}$ against ALL reflections. The weighted $R$-factor $w R$ and goodness of fit $S$ are based on $F^{2}$, conventional $R$-factors $R$ are based on $F$, with $F$ set to zero for negative $F^{2}$. The threshold expression of $F^{2}>\sigma\left(F^{2}\right)$ is used only for calculating $R$-factors(gt) $e t c$. and is not relevant to the choice of reflections for refinement. $R$-factors based on $F^{2}$ are statistically about twice as large as those based on $F$, and $R$ - factors based on ALL data will be even larger.

Fractional atomic coordinates and isotropic or equivalent isotropic displacement parameters $\left(\AA^{2}\right)$

\begin{tabular}{lllll}
\hline & $x$ & $y$ & $z$ & $U_{\text {iso }} * / U_{\text {eq }}$ \\
\hline Fe1 & $0.87669(12)$ & $1.09597(7)$ & $0.19524(6)$ & $0.0333(3)$ \\
Fe2 & $1.07650(12)$ & $0.91292(7)$ & $0.24157(6)$ & $0.0341(3)$ \\
P1 & $0.6388(2)$ & $1.21710(14)$ & $0.18129(12)$ & $0.0316(5)$
\end{tabular}




\begin{tabular}{|c|c|c|c|c|}
\hline $\mathrm{P} 2$ & $1.1691(2)$ & $0.74053(13)$ & $0.30622(12)$ & $0.0314(5)$ \\
\hline S1 & $0.9131(2)$ & 0.99887 (13) & $0.32702(11)$ & $0.0359(5)$ \\
\hline S2 & $0.8517(2)$ & $0.94621(13)$ & $0.16626(12)$ & $0.0356(5)$ \\
\hline $\mathrm{O} 1$ & $0.9526(7)$ & $1.1484(4)$ & 0.0249 (3) & $0.0531(16)$ \\
\hline $\mathrm{O} 2$ & $1.0428(6)$ & $1.2238(4)$ & $0.2488(3)$ & $0.0423(14)$ \\
\hline $\mathrm{O} 3$ & $1.2386(6)$ & $0.9101(4)$ & $0.0911(3)$ & $0.0574(17)$ \\
\hline $\mathrm{O} 4$ & $1.3209(6)$ & $0.9648(4)$ & $0.3122(3)$ & $0.0482(15)$ \\
\hline $\mathrm{C} 1$ & $0.9215(8)$ & $1.1291(5)$ & $0.0921(5)$ & $0.035(2)$ \\
\hline $\mathrm{C} 2$ & $0.9700(9)$ & $1.1748(5)$ & 0.2264 (4) & $0.037(2)$ \\
\hline $\mathrm{C} 3$ & $1.1720(8)$ & $0.9076(5)$ & 0.1509 (5) & $0.040(2)$ \\
\hline $\mathrm{C} 4$ & $1.2219(9)$ & $0.9448(5)$ & $0.2834(4)$ & $0.0342(19)$ \\
\hline $\mathrm{C} 5$ & $0.7706(8)$ & $0.9347(5)$ & $0.3615(4)$ & $0.038(2)$ \\
\hline $\mathrm{H} 5 \mathrm{~A}$ & 0.8029 & 0.8936 & 0.4183 & $0.045^{*}$ \\
\hline H5B & 0.6712 & 0.9921 & 0.3632 & $0.045 *$ \\
\hline C6 & $0.7389(8)$ & $0.8584(5)$ & $0.3127(5)$ & $0.039(2)$ \\
\hline H6A & 0.8317 & 0.7935 & 0.3190 & $0.047^{*}$ \\
\hline H6B & 0.6523 & 0.8353 & 0.3362 & $0.047^{*}$ \\
\hline C7 & $0.6992(8)$ & $0.9069(5)$ & $0.2232(5)$ & $0.040(2)$ \\
\hline H7A & 0.6056 & 0.9712 & 0.2172 & $0.049 *$ \\
\hline H7B & 0.6725 & 0.8545 & 0.1978 & $0.049^{*}$ \\
\hline $\mathrm{C} 8$ & $0.6245(8)$ & $1.3568(5)$ & $0.1372(4)$ & $0.0308(18)$ \\
\hline C9 & $0.7434(9)$ & $1.3833(5)$ & $0.0962(5)$ & $0.037(2)$ \\
\hline H9 & 0.8371 & 1.3271 & 0.0938 & $0.045^{*}$ \\
\hline $\mathrm{C} 10$ & $0.7330(9)$ & $1.4864(5)$ & 0.0589 (4) & $0.042(2)$ \\
\hline H10 & 0.8180 & 1.5017 & 0.0323 & $0.051^{*}$ \\
\hline C11 & $0.5929(9)$ & $1.5688(5)$ & $0.0612(4)$ & $0.039(2)$ \\
\hline H11 & 0.5804 & 1.6408 & 0.0343 & $0.047^{*}$ \\
\hline $\mathrm{C} 12$ & $0.4745(9)$ & $1.5446(6)$ & $0.1024(5)$ & $0.043(2)$ \\
\hline H12 & 0.3808 & 1.6006 & 0.1051 & $0.051^{*}$ \\
\hline $\mathrm{C} 13$ & $0.4893(9)$ & $1.4402(5)$ & $0.1400(4)$ & $0.044(2)$ \\
\hline H13 & 0.4055 & 1.4253 & 0.1682 & $0.052^{*}$ \\
\hline C14 & $0.4933(8)$ & $1.2007(5)$ & $0.1161(5)$ & 0.0325 (19) \\
\hline $\mathrm{C} 15$ & $0.5438(8)$ & $1.1447(5)$ & $0.0520(4)$ & $0.036(2)$ \\
\hline H15 & 0.6512 & 1.1110 & 0.0473 & $0.043^{*}$ \\
\hline $\mathrm{C} 16$ & $0.4372(8)$ & $1.1397(5)$ & $-0.0023(4)$ & $0.0322(18)$ \\
\hline H16 & 0.4730 & 1.1051 & -0.0459 & $0.039^{*}$ \\
\hline $\mathrm{C} 17$ & $0.2815(9)$ & $1.1824(5)$ & $0.0038(5)$ & $0.042(2)$ \\
\hline H17 & 0.2095 & 1.1747 & -0.0330 & $0.050^{*}$ \\
\hline C18 & $0.2319(9)$ & $1.2374(5)$ & $0.0653(5)$ & $0.041(2)$ \\
\hline H18 & 0.1240 & 1.2693 & 0.0701 & $0.049 *$ \\
\hline C19 & $0.3360(8)$ & $1.2466(5)$ & $0.1195(5)$ & $0.040(2)$ \\
\hline H19 & 0.2983 & 1.2857 & 0.1605 & $0.048^{*}$ \\
\hline $\mathrm{C} 20$ & $0.5427(8)$ & $1.2287(5)$ & 0.2775 (4) & $0.0261(17)$ \\
\hline $\mathrm{C} 21$ & $0.5778(9)$ & $1.2937(5)$ & $0.3258(5)$ & $0.043(2)$ \\
\hline $\mathrm{H} 21$ & 0.6423 & 1.3355 & 0.3065 & $0.051^{*}$ \\
\hline $\mathrm{C} 22$ & $0.5154(9)$ & $1.2951(6)$ & $0.4027(5)$ & $0.044(2)$ \\
\hline $\mathrm{H} 22$ & 0.5350 & 1.3399 & 0.4350 & $0.053^{*}$ \\
\hline $\mathrm{C} 23$ & $0.4285(9)$ & $1.2335(6)$ & $0.4304(5)$ & $0.044(2)$ \\
\hline
\end{tabular}




$\begin{array}{lllll}\mathrm{H} 23 & 0.3891 & 1.2346 & 0.4829 & 0.053^{*} \\ \mathrm{C} 24 & 0.3951(8) & 1.1701(5) & 0.3862(5) & 0.042(2) \\ \mathrm{H} 24 & 0.3334 & 1.1268 & 0.4073 & 0.051^{*} \\ \mathrm{C} 25 & 0.4529(9) & 1.1688(5) & 0.3083(5) & 0.041(2) \\ \mathrm{H} 25 & 0.4284 & 1.1250 & 0.2766 & 0.049^{*} \\ \mathrm{C} 26 & 1.1264(8) & 0.6384(5) & 0.2631(4) & 0.0314(18) \\ \mathrm{C} 27 & 1.0331(9) & 0.6666(5) & 0.1930(5) & 0.037(2) \\ \mathrm{H} 27 & 0.9904 & 0.7404 & 0.1671 & 0.045^{*} \\ \mathrm{C} 28 & 0.9989(8) & 0.5915(5) & 0.1585(5) & 0.039(2) \\ \mathrm{H} 28 & 0.9361 & 0.6139 & 0.1090 & 0.047^{*} \\ \mathrm{C} 29 & 1.0557(8) & 0.4852(5) & 0.1959(5) & 0.037(2) \\ \mathrm{H} 29 & 1.0286 & 0.4332 & 0.1744 & 0.044^{*} \\ \mathrm{C} 30 & 1.1545(8) & 0.4541(5) & 0.2664(4) & 0.037(2) \\ \mathrm{H} 30 & 1.1975 & 0.3801 & 0.2917 & 0.044^{*} \\ \mathrm{C} 31 & 1.1907(8) & 0.5291(5) & 0.2998(4) & 0.0325(19) \\ \mathrm{H} 31 & 1.2587 & 0.5067 & 0.3474 & 0.039^{*} \\ \mathrm{C} 32 & 1.1094(8) & 0.7174(5) & 0.4129(4) & 0.0304(19) \\ \mathrm{C} 33 & 1.0258(8) & 0.6510(5) & 0.4409(4) & 0.0336(19) \\ \mathrm{H} 33 & 1.0012 & 0.6101 & 0.4066 & 0.040^{*} \\ \mathrm{C} 34 & 0.9755(8) & 0.6434(5) & 0.5219(5) & 0.037(2) \\ \mathrm{H} 34 & 0.9205 & 0.5955 & 0.5428 & 0.044^{*} \\ \mathrm{C} 35 & 1.0078(9) & 0.7065(5) & 0.5695(4) & 0.040(2) \\ \mathrm{H} 35 & 0.9710 & 0.7038 & 0.6227 & 0.048^{*} \\ \mathrm{C} 36 & 1.0909(8) & 0.7721(6) & 0.5418(5) & 0.041(2) \\ \mathrm{H} 36 & 1.1132 & 0.8140 & 0.5760 & 0.049^{*} \\ \mathrm{C} 37 & 1.1437(8) & 0.7786(5) & 0.4640(5) & 0.037(2) \\ \mathrm{H} 37 & 1.2029 & 0.8243 & 0.4450 & 0.044^{*} \\ \mathrm{C} 38 & 1.3813(8) & 0.6816(5) & 0.3197(4) & 0.0302(18) \\ \mathrm{C} 39 & 1.4600(9) & 0.6203(5) & 0.3913(5) & 0.044(2) \\ \mathrm{H} 39 & 1.4042 & 0.6103 & 0.4387 & 0.053^{*} \\ \mathrm{C} 40 & 1.6209(10) & 0.5719(6) & 0.3968(5) & 0.051(2) \\ \mathrm{H} 40 & 1.6742 & 0.5324 & 0.4478 & 0.061^{*} \\ \mathrm{C} 41 & 1.7028(9) & 0.5819(5) & 0.3263(5) & 0.047(2) \\ \mathrm{H} 41 & 1.8116 & 0.5475 & 0.3283 & 0.056^{*} \\ \mathrm{C} 42 & 1.6247(9) & 0.6408(5) & 0.2565(5) & 0.042(2) \\ \mathrm{H} 42 & 1.6801 & 0.6487 & 0.2087 & 0.050^{*} \\ \mathrm{C} 43 & 1.4680(9) & 0.6905(5) & 0.2509(5) & 0.038(2) \\ \mathrm{H} 43 & 1.4172 & 0.7316 & 0.1996 & 0.045^{*} \\ & & & & \end{array}$

Atomic displacement parameters $\left(\AA^{2}\right)$

\begin{tabular}{lllllll}
\hline & $U^{11}$ & $U^{22}$ & $U^{33}$ & $U^{12}$ & $U^{13}$ & $U^{23}$ \\
\hline Fe1 & $0.0382(7)$ & $0.0381(6)$ & $0.0270(7)$ & $-0.0102(5)$ & $0.0135(5)$ & $-0.0182(5)$ \\
Fe2 & $0.0353(7)$ & $0.0404(6)$ & $0.0284(7)$ & $-0.0078(5)$ & $0.0130(5)$ & $-0.0191(5)$ \\
P1 & $0.0332(12)$ & $0.0366(10)$ & $0.0252(12)$ & $-0.0072(9)$ & $0.0094(10)$ & $-0.0142(9)$ \\
P2 & $0.0324(12)$ & $0.0381(10)$ & $0.0254(12)$ & $-0.0074(9)$ & $0.0083(9)$ & $-0.0177(9)$ \\
S1 & $0.0384(12)$ & $0.0419(11)$ & $0.0294(12)$ & $-0.0086(9)$ & $0.0137(10)$ & $-0.0201(9)$ \\
S2 & $0.0384(12)$ & $0.0385(10)$ & $0.0311(13)$ & $-0.0080(9)$ & $0.0100(10)$ & $-0.0181(9)$
\end{tabular}




\begin{tabular}{|c|c|c|c|c|c|c|}
\hline $\mathrm{O} 1$ & $0.062(4)$ & $0.068(4)$ & $0.036(4)$ & $-0.026(3)$ & 0.024 & $-0.020(3)$ \\
\hline $\mathrm{O} 2$ & $0.050(4)$ & $0.054(3)$ & $0.033(3)$ & $-0.024(3)$ & $0.014(3)$ & $-0.022(3)$ \\
\hline $\mathrm{O} 3$ & $0.044(4)$ & 0.099 (4) & 0.033 (4) & $-0.020(3)$ & $0.023(3)$ & $-0.028(3)$ \\
\hline $\mathrm{O} 4$ & 0.039 (4) & $0.056(3)$ & 0.049 (4) & $-0.014(3)$ & 0.009 (3) & $-0.013(3)$ \\
\hline $\mathrm{C} 1$ & $0.033(5)$ & $0.034(4)$ & $0.044(6)$ & $-0.014(4)$ & $0.005(4)$ & $-0.014(4)$ \\
\hline $\mathrm{C} 2$ & $0.045(5)$ & $0.037(4)$ & $0.026(5)$ & $-0.013(4)$ & 0.019 (4) & -0.005 (4) \\
\hline C3 & $0.028(5)$ & $0.049(5)$ & $0.042(6)$ & $-0.010(4)$ & 0.008 (4) & $-0.016(4)$ \\
\hline $\mathrm{C} 4$ & $0.030(5)$ & $0.042(4)$ & $0.028(5)$ & -0.007 (4) & 0.017 (4) & -0.009 (4) \\
\hline C5 & $0.031(4)$ & $0.041(4)$ & $0.029(5)$ & $0.002(4)$ & $0.020(4)$ & $-0.006(4)$ \\
\hline C6 & $0.033(5)$ & $0.035(4)$ & $0.045(6)$ & $-0.006(4)$ & 0.010 (4) & $-0.007(4)$ \\
\hline $\mathrm{C} 7$ & $0.048(5)$ & $0.022(4)$ & $0.057(6)$ & $-0.010(4)$ & $0.005(5)$ & $-0.023(4)$ \\
\hline $\mathrm{C} 8$ & $0.033(5)$ & $0.036(4)$ & $0.025(5)$ & -0.009 (4) & 0.005 (4) & $-0.016(3)$ \\
\hline C9 & $0.040(5)$ & $0.030(4)$ & $0.044(6)$ & -0.010 & $0.012(4)$ & $-0.016(4)$ \\
\hline $\mathrm{C} 10$ & $0.045(5)$ & $0.057(5)$ & $0.034(5)$ & -0.024 & $0.015(4)$ & -0.018 \\
\hline $\mathrm{C} 11$ & $0.056(6)$ & $0.034(4)$ & $0.026(5)$ & -0.014 & $0.003(4)$ & $0.000(4)$ \\
\hline $\mathrm{C} 12$ & $0.049(6)$ & $0.045(5)$ & $0.030(5)$ & -0.005 (4) & $-0.001(4)$ & $-0.017(4)$ \\
\hline C13 & $0.048(6)$ & $0.041(4)$ & $0.037(5)$ & -0.005 (4) & $0.014(4)$ & -0.013 \\
\hline $\mathrm{C} 14$ & $0.034(5)$ & $0.029(4)$ & $0.038(5)$ & -0.011 & 0.009 (4) & $-0.012(3)$ \\
\hline $\mathrm{C} 15$ & $0.032(5)$ & $0.038(4)$ & $0.030(5)$ & $0.002(4)$ & 0.007 (4) & $-0.016(4)$ \\
\hline $\mathrm{C} 16$ & $0.034(5)$ & $0.037(4)$ & $0.025(5)$ & -0.007 (4) & $0.012(4)$ & $-0.015(3)$ \\
\hline $\mathrm{C} 17$ & $0.042(5)$ & $0.049(5)$ & $0.044(6)$ & -0.020 & 0.002 (4) & $-0.023(4)$ \\
\hline $\mathrm{C} 18$ & $0.029(5)$ & $0.064(5)$ & $0.043(6)$ & -0.023 & $0.020(4)$ & -0.029 (4) \\
\hline $\mathrm{C} 19$ & $0.036(5)$ & $0.043(4)$ & $0.054(6)$ & -0.018 & $0.025(4)$ & $-0.032(4)$ \\
\hline $\mathrm{C} 20$ & $0.026(4)$ & $0.026(4)$ & $0.027(5)$ & -0.008 & 0.009 (4) & -0.009 (3) \\
\hline $\mathrm{C} 21$ & $0.050(5)$ & $0.041(4)$ & $0.038(6)$ & $-0.012(4)$ & $0.016(4)$ & $-0.017(4)$ \\
\hline $\mathrm{C} 22$ & $0.043(5)$ & $0.063(5)$ & $0.032(5)$ & -0.014 & 0.008 (4) & -0.029 (4) \\
\hline $\mathrm{C} 23$ & $0.045(6)$ & $0.055(5)$ & $0.023(5)$ & -0.003 & $0.012(4)$ & $-0.013(4)$ \\
\hline C24 & $0.038(5)$ & $0.036(4)$ & $0.038(6)$ & $0.002(4)$ & $0.021(4)$ & -0.001 \\
\hline $\mathrm{C} 25$ & $0.044(5)$ & 0.035 (4) & $0.029(5)$ & $0.009(4)$ & $0.006(4)$ & $-0.012(4)$ \\
\hline $\mathrm{C} 26$ & $0.035(5)$ & $0.034(4)$ & $0.027(5)$ & $-0.007(4)$ & $0.022(4)$ & -0.019 \\
\hline $\mathrm{C} 27$ & $0.043(5)$ & $0.032(4)$ & $0.038(5)$ & $-0.006(4)$ & $0.016(4)$ & $-0.021(4)$ \\
\hline C28 & $0.037(5)$ & $0.046(4)$ & $0.040(5)$ & -0.013 & $0.013(4)$ & -0.023 \\
\hline $\mathrm{C} 29$ & $0.038(5)$ & $0.036(4)$ & $0.044(6)$ & -0.013 & $0.018(4)$ & -0.022 \\
\hline $\mathrm{C} 30$ & $0.040(5)$ & $0.035(4)$ & $0.032(5)$ & $-0.002(4)$ & $0.015(4)$ & $-0.017(4)$ \\
\hline C31 & $0.028(4)$ & $0.049(4)$ & $0.024(5)$ & -0.010 & 0.008 (4) & $-0.021(4)$ \\
\hline $\mathrm{C} 32$ & 0.019 (4) & $0.035(4)$ & $0.033(5)$ & $0.003(3)$ & $-0.002(4)$ & $-0.016(4)$ \\
\hline $\mathrm{C} 33$ & $0.030(5)$ & $0.033(4)$ & $0.032(5)$ & $0.000(4)$ & $0.003(4)$ & -0.013 \\
\hline $\mathrm{C} 34$ & $0.034(5)$ & $0.033(4)$ & $0.034(5)$ & -0.001 & 0.017 (4) & $-0.004(4)$ \\
\hline $\mathrm{C} 35$ & $0.043(5)$ & $0.055(5)$ & $0.022(5)$ & -0.011 & $0.018(4)$ & $-0.017(4)$ \\
\hline $\mathrm{C} 36$ & $0.037(5)$ & $0.060(5)$ & $0.031(5)$ & -0.011 & $0.016(4)$ & $-0.027(4)$ \\
\hline C37 & $0.034(5)$ & $0.038(4)$ & $0.037(5)$ & -0.005 & $0.003(4)$ & $-0.016(4)$ \\
\hline $\mathrm{C} 38$ & $0.041(5)$ & $0.032(4)$ & $0.020(5)$ & -0.014 & $0.013(4)$ & $-0.009(3)$ \\
\hline C39 & $0.036(5)$ & $0.053(5)$ & $0.043(6)$ & $-0.012(4)$ & $0.014(4)$ & -0.015 \\
\hline $\mathrm{C} 40$ & $0.050(6)$ & $0.063(5)$ & $0.037(6)$ & $-0.008(5)$ & $-0.004(5)$ & -0.019 (4) \\
\hline $\mathrm{C} 41$ & $0.037(5)$ & $0.050(5)$ & $0.052(6)$ & $-0.002(4)$ & $0.014(5)$ & $-0.028(5)$ \\
\hline $\mathrm{C} 42$ & $0.043(5)$ & $0.050(5)$ & $0.033(5)$ & -0.013 & $0.014(4)$ & -0.015 \\
\hline $\mathrm{C} 43$ & $0.042(5)$ & $0.045(4)$ & $0.023(5)$ & -0.009 (4) & $0.002(4)$ & -0.010 \\
\hline
\end{tabular}


Geometric parameters $\left(\AA,{ }^{\circ}\right)$

\begin{tabular}{|c|c|c|c|}
\hline $\mathrm{Fe} 1-\mathrm{C} 2$ & $1.719(8)$ & $\mathrm{C} 17-\mathrm{H} 17$ & 0.9500 \\
\hline $\mathrm{Fe} 1-\mathrm{C} 1$ & $1.773(8)$ & $\mathrm{C} 18-\mathrm{C} 19$ & $1.374(9)$ \\
\hline $\mathrm{Fe} 1-\mathrm{P} 1$ & $2.237(2)$ & C18-H18 & 0.9500 \\
\hline $\mathrm{Fe} 1-\mathrm{S} 2$ & $2.254(2)$ & C19-H19 & 0.9500 \\
\hline $\mathrm{Fe} 1-\mathrm{S} 1$ & $2.285(2)$ & $\mathrm{C} 20-\mathrm{C} 25$ & $1.355(9)$ \\
\hline $\mathrm{Fe} 1-\mathrm{Fe} 2$ & $2.5167(16)$ & $\mathrm{C} 20-\mathrm{C} 21$ & $1.419(9)$ \\
\hline $\mathrm{Fe} 2-\mathrm{C} 4$ & $1.720(8)$ & $\mathrm{C} 21-\mathrm{C} 22$ & $1.408(9)$ \\
\hline $\mathrm{Fe} 2-\mathrm{C} 3$ & $1.750(8)$ & $\mathrm{C} 21-\mathrm{H} 21$ & 0.9500 \\
\hline $\mathrm{Fe} 2-\mathrm{P} 2$ & $2.230(2)$ & $\mathrm{C} 22-\mathrm{C} 23$ & $1.339(10)$ \\
\hline $\mathrm{Fe} 2-\mathrm{S} 1$ & $2.276(2)$ & $\mathrm{C} 22-\mathrm{H} 22$ & 0.9500 \\
\hline $\mathrm{Fe} 2-\mathrm{S} 2$ & $2.287(2)$ & $\mathrm{C} 23-\mathrm{C} 24$ & $1.349(9)$ \\
\hline $\mathrm{P} 1-\mathrm{C} 14$ & $1.826(7)$ & $\mathrm{C} 23-\mathrm{H} 23$ & 0.9500 \\
\hline $\mathrm{P} 1-\mathrm{C} 8$ & $1.834(7)$ & $\mathrm{C} 24-\mathrm{C} 25$ & $1.407(9)$ \\
\hline $\mathrm{P} 1-\mathrm{C} 20$ & $1.843(7)$ & $\mathrm{C} 24-\mathrm{H} 24$ & 0.9500 \\
\hline $\mathrm{P} 2-\mathrm{C} 26$ & $1.829(7)$ & $\mathrm{C} 25-\mathrm{H} 25$ & 0.9500 \\
\hline $\mathrm{P} 2-\mathrm{C} 38$ & $1.839(7)$ & $\mathrm{C} 26-\mathrm{C} 27$ & $1.369(9)$ \\
\hline $\mathrm{P} 2-\mathrm{C} 32$ & $1.859(8)$ & $\mathrm{C} 26-\mathrm{C} 31$ & $1.399(8)$ \\
\hline $\mathrm{S} 1-\mathrm{C} 5$ & $1.812(7)$ & $\mathrm{C} 27-\mathrm{C} 28$ & $1.382(8)$ \\
\hline $\mathrm{S} 2-\mathrm{C} 7$ & $1.837(8)$ & $\mathrm{C} 27-\mathrm{H} 27$ & 0.9500 \\
\hline $\mathrm{O} 1-\mathrm{C} 1$ & $1.156(8)$ & $\mathrm{C} 28-\mathrm{C} 29$ & $1.365(8)$ \\
\hline $\mathrm{O} 2-\mathrm{C} 2$ & $1.189(8)$ & $\mathrm{C} 28-\mathrm{H} 28$ & 0.9500 \\
\hline $\mathrm{O} 3-\mathrm{C} 3$ & $1.171(9)$ & $\mathrm{C} 29-\mathrm{C} 30$ & $1.398(9)$ \\
\hline $\mathrm{O} 4-\mathrm{C} 4$ & $1.160(8)$ & $\mathrm{C} 29-\mathrm{H} 29$ & 0.9500 \\
\hline $\mathrm{C} 5-\mathrm{C} 6$ & $1.538(8)$ & $\mathrm{C} 30-\mathrm{C} 31$ & $1.380(8)$ \\
\hline $\mathrm{C} 5-\mathrm{H} 5 \mathrm{~A}$ & 0.9900 & $\mathrm{C} 30-\mathrm{H} 30$ & 0.9500 \\
\hline $\mathrm{C} 5-\mathrm{H} 5 \mathrm{~B}$ & 0.9900 & $\mathrm{C} 31-\mathrm{H} 31$ & 0.9500 \\
\hline $\mathrm{C} 6-\mathrm{C} 7$ & $1.502(9)$ & $\mathrm{C} 32-\mathrm{C} 33$ & $1.366(9)$ \\
\hline $\mathrm{C} 6-\mathrm{H} 6 \mathrm{~A}$ & 0.9900 & $\mathrm{C} 32-\mathrm{C} 37$ & $1.409(9)$ \\
\hline C6- $-\mathrm{H} 6 \mathrm{~B}$ & 0.9900 & $\mathrm{C} 33-\mathrm{C} 34$ & $1.425(9)$ \\
\hline C7-H7A & 0.9900 & C33-H33 & 0.9500 \\
\hline C7-H7B & 0.9900 & $\mathrm{C} 34-\mathrm{C} 35$ & $1.380(9)$ \\
\hline $\mathrm{C} 8-\mathrm{C} 9$ & $1.384(9)$ & C34-H34 & 0.9500 \\
\hline $\mathrm{C} 8-\mathrm{C} 13$ & $1.385(9)$ & $\mathrm{C} 35-\mathrm{C} 36$ & $1.352(9)$ \\
\hline $\mathrm{C} 9-\mathrm{C} 10$ & $1.372(8)$ & C35-H35 & 0.9500 \\
\hline C9-H9 & 0.9500 & $\mathrm{C} 36-\mathrm{C} 37$ & $1.384(9)$ \\
\hline $\mathrm{C} 10-\mathrm{C} 11$ & $1.405(9)$ & C36- & 0.9500 \\
\hline $\mathrm{C} 10-\mathrm{H} 10$ & 0.9500 & C37-H37 & 0.9500 \\
\hline $\mathrm{C} 11-\mathrm{C} 12$ & $1.369(10)$ & $\mathrm{C} 38-\mathrm{C} 39$ & $1.362(9)$ \\
\hline $\mathrm{C} 11-\mathrm{H} 11$ & 0.9500 & $\mathrm{C} 38-\mathrm{C} 43$ & $1.402(9)$ \\
\hline $\mathrm{C} 12-\mathrm{C} 13$ & $1.378(9)$ & $\mathrm{C} 39-\mathrm{C} 40$ & $1.398(10)$ \\
\hline $\mathrm{C} 12-\mathrm{H} 12$ & 0.9500 & C39-H39 & 0.9500 \\
\hline C13-H13 & 0.9500 & $\mathrm{C} 40-\mathrm{C} 41$ & $1.400(10)$ \\
\hline $\mathrm{C} 14-\mathrm{C} 19$ & $1.376(9)$ & $\mathrm{C} 40-\mathrm{H} 40$ & 0.9500 \\
\hline $\mathrm{C} 14-\mathrm{C} 15$ & $1.432(9)$ & $\mathrm{C} 41-\mathrm{C} 42$ & $1.329(10)$ \\
\hline $\mathrm{C} 15-\mathrm{C} 16$ & $1.367(9)$ & $\mathrm{C} 41-\mathrm{H} 41$ & 0.9500 \\
\hline C15-H15 & 0.9500 & $\mathrm{C} 42-\mathrm{C} 43$ & $1.366(9)$ \\
\hline
\end{tabular}




\begin{tabular}{|c|c|c|c|}
\hline $\mathrm{C} 16-\mathrm{C} 17$ & $1.364(9)$ & $\mathrm{C} 42-\mathrm{H} 42$ & 0.9500 \\
\hline $\mathrm{C} 16-\mathrm{H} 16$ & 0.9500 & $\mathrm{C} 43-\mathrm{H} 43$ & 0.9500 \\
\hline $\mathrm{C} 17-\mathrm{C} 18$ & $1.387(9)$ & & \\
\hline $\mathrm{C} 2-\mathrm{Fe} 1-\mathrm{C} 1$ & $93.2(3)$ & $\mathrm{C} 19-\mathrm{C} 14-\mathrm{P} 1$ & $124.3(6)$ \\
\hline $\mathrm{C} 2-\mathrm{Fe} 1-\mathrm{P} 1$ & $96.3(2)$ & $\mathrm{C} 15-\mathrm{C} 14-\mathrm{P} 1$ & $118.7(6)$ \\
\hline $\mathrm{C} 1-\mathrm{Fe} 1-\mathrm{P} 1$ & $96.4(2)$ & $\mathrm{C} 16-\mathrm{C} 15-\mathrm{C} 14$ & $119.9(7)$ \\
\hline $\mathrm{C} 2-\mathrm{Fe} 1-\mathrm{S} 2$ & $156.9(2)$ & $\mathrm{C} 16-\mathrm{C} 15-\mathrm{H} 15$ & 120.0 \\
\hline $\mathrm{C} 1-\mathrm{Fe} 1-\mathrm{S} 2$ & $86.5(2)$ & $\mathrm{C} 14-\mathrm{C} 15-\mathrm{H} 15$ & 120.0 \\
\hline $\mathrm{P} 1-\mathrm{Fe} 1-\mathrm{S} 2$ & $106.71(9)$ & $\mathrm{C} 17-\mathrm{C} 16-\mathrm{C} 15$ & $122.5(7)$ \\
\hline $\mathrm{C} 2-\mathrm{Fe} 1-\mathrm{S} 1$ & $87.4(2)$ & $\mathrm{C} 17-\mathrm{C} 16-\mathrm{H} 16$ & 118.7 \\
\hline $\mathrm{C} 1-\mathrm{Fe} 1-\mathrm{S} 1$ & $156.3(2)$ & $\mathrm{C} 15-\mathrm{C} 16-\mathrm{H} 16$ & 118.7 \\
\hline $\mathrm{P} 1-\mathrm{Fe} 1-\mathrm{S} 1$ & $107.06(8)$ & $\mathrm{C} 16-\mathrm{C} 17-\mathrm{C} 18$ & $117.8(7)$ \\
\hline $\mathrm{S} 2-\mathrm{Fe} 1-\mathrm{S} 1$ & $83.84(8)$ & $\mathrm{C} 16-\mathrm{C} 17-\mathrm{H} 17$ & 121.1 \\
\hline $\mathrm{C} 2-\mathrm{Fe} 1-\mathrm{Fe} 2$ & $100.6(2)$ & $\mathrm{C} 18-\mathrm{C} 17-\mathrm{H} 17$ & 121.1 \\
\hline $\mathrm{C} 1-\mathrm{Fe} 1-\mathrm{Fe} 2$ & $100.5(2)$ & $\mathrm{C} 19-\mathrm{C} 18-\mathrm{C} 17$ & $121.1(7)$ \\
\hline $\mathrm{P} 1-\mathrm{Fe} 1-\mathrm{Fe} 2$ & $155.34(8)$ & $\mathrm{C} 19-\mathrm{C} 18-\mathrm{H} 18$ & 119.4 \\
\hline $\mathrm{S} 2-\mathrm{Fe} 1-\mathrm{Fe} 2$ & $56.98(6)$ & $\mathrm{C} 17-\mathrm{C} 18-\mathrm{H} 18$ & 119.4 \\
\hline $\mathrm{S} 1-\mathrm{Fe} 1-\mathrm{Fe} 2$ & $56.33(6)$ & $\mathrm{C} 18-\mathrm{C} 19-\mathrm{C} 14$ & $121.8(7)$ \\
\hline $\mathrm{C} 4-\mathrm{Fe} 2-\mathrm{C} 3$ & $90.2(3)$ & $\mathrm{C} 18-\mathrm{C} 19-\mathrm{H} 19$ & 119.1 \\
\hline $\mathrm{C} 4-\mathrm{Fe} 2-\mathrm{P} 2$ & $93.3(2)$ & $\mathrm{C} 14-\mathrm{C} 19-\mathrm{H} 19$ & 119.1 \\
\hline $\mathrm{C} 3-\mathrm{Fe} 2-\mathrm{P} 2$ & $100.2(2)$ & $\mathrm{C} 25-\mathrm{C} 20-\mathrm{C} 21$ & $118.4(7)$ \\
\hline $\mathrm{C} 4-\mathrm{Fe} 2-\mathrm{S} 1$ & $88.2(2)$ & $\mathrm{C} 25-\mathrm{C} 20-\mathrm{P} 1$ & $122.5(5)$ \\
\hline $\mathrm{C} 3-\mathrm{Fe} 2-\mathrm{S} 1$ & $153.7(2)$ & $\mathrm{C} 21-\mathrm{C} 20-\mathrm{P} 1$ & $118.7(6)$ \\
\hline $\mathrm{P} 2-\mathrm{Fe} 2-\mathrm{S} 1$ & $106.10(9)$ & $\mathrm{C} 22-\mathrm{C} 21-\mathrm{C} 20$ & $118.8(7)$ \\
\hline $\mathrm{C} 4-\mathrm{Fe} 2-\mathrm{S} 2$ & $156.0(2)$ & $\mathrm{C} 22-\mathrm{C} 21-\mathrm{H} 21$ & 120.6 \\
\hline $\mathrm{C} 3-\mathrm{Fe} 2-\mathrm{S} 2$ & $87.6(3)$ & $\mathrm{C} 20-\mathrm{C} 21-\mathrm{H} 21$ & 120.6 \\
\hline $\mathrm{P} 2-\mathrm{Fe} 2-\mathrm{S} 2$ & $110.61(8)$ & $\mathrm{C} 23-\mathrm{C} 22-\mathrm{C} 21$ & $120.3(7)$ \\
\hline $\mathrm{S} 1-\mathrm{Fe} 2-\mathrm{S} 2$ & $83.30(8)$ & $\mathrm{C} 23-\mathrm{C} 22-\mathrm{H} 22$ & 119.8 \\
\hline $\mathrm{C} 4-\mathrm{Fe} 2-\mathrm{Fe} 1$ & $101.1(2)$ & $\mathrm{C} 21-\mathrm{C} 22-\mathrm{H} 22$ & 119.8 \\
\hline $\mathrm{C} 3-\mathrm{Fe} 2-\mathrm{Fe} 1$ & $98.0(2)$ & $\mathrm{C} 22-\mathrm{C} 23-\mathrm{C} 24$ & $122.0(8)$ \\
\hline $\mathrm{P} 2-\mathrm{Fe} 2-\mathrm{Fe} 1$ & $156.70(8)$ & $\mathrm{C} 22-\mathrm{C} 23-\mathrm{H} 23$ & 119.0 \\
\hline $\mathrm{S} 1-\mathrm{Fe} 2-\mathrm{Fe} 1$ & $56.69(6)$ & $\mathrm{C} 24-\mathrm{C} 23-\mathrm{H} 23$ & 119.0 \\
\hline $\mathrm{S} 2-\mathrm{Fe} 2-\mathrm{Fe} 1$ & $55.71(6)$ & $\mathrm{C} 23-\mathrm{C} 24-\mathrm{C} 25$ & $119.0(8)$ \\
\hline $\mathrm{C} 14-\mathrm{P} 1-\mathrm{C} 8$ & $100.7(3)$ & $\mathrm{C} 23-\mathrm{C} 24-\mathrm{H} 24$ & 120.5 \\
\hline $\mathrm{C} 14-\mathrm{P} 1-\mathrm{C} 20$ & $103.1(3)$ & $\mathrm{C} 25-\mathrm{C} 24-\mathrm{H} 24$ & 120.5 \\
\hline $\mathrm{C} 8-\mathrm{P} 1-\mathrm{C} 20$ & $102.0(3)$ & $\mathrm{C} 20-\mathrm{C} 25-\mathrm{C} 24$ & $121.5(7)$ \\
\hline $\mathrm{C} 14-\mathrm{P} 1-\mathrm{Fe} 1$ & $117.7(2)$ & $\mathrm{C} 20-\mathrm{C} 25-\mathrm{H} 25$ & 119.3 \\
\hline $\mathrm{C} 8-\mathrm{P} 1-\mathrm{Fe} 1$ & $116.2(2)$ & $\mathrm{C} 24-\mathrm{C} 25-\mathrm{H} 25$ & 119.3 \\
\hline $\mathrm{C} 20-\mathrm{P} 1-\mathrm{Fe} 1$ & $114.9(2)$ & $\mathrm{C} 27-\mathrm{C} 26-\mathrm{C} 31$ & $118.1(6)$ \\
\hline $\mathrm{C} 26-\mathrm{P} 2-\mathrm{C} 38$ & $99.4(3)$ & $\mathrm{C} 27-\mathrm{C} 26-\mathrm{P} 2$ & $120.9(5)$ \\
\hline $\mathrm{C} 26-\mathrm{P} 2-\mathrm{C} 32$ & $104.5(3)$ & $\mathrm{C} 31-\mathrm{C} 26-\mathrm{P} 2$ & $121.0(6)$ \\
\hline $\mathrm{C} 38-\mathrm{P} 2-\mathrm{C} 32$ & $102.6(3)$ & $\mathrm{C} 26-\mathrm{C} 27-\mathrm{C} 28$ & $122.5(6)$ \\
\hline $\mathrm{C} 26-\mathrm{P} 2-\mathrm{Fe} 2$ & $119.9(2)$ & $\mathrm{C} 26-\mathrm{C} 27-\mathrm{H} 27$ & 118.8 \\
\hline $\mathrm{C} 38-\mathrm{P} 2-\mathrm{Fe} 2$ & $115.9(2)$ & $\mathrm{C} 28-\mathrm{C} 27-\mathrm{H} 27$ & 118.8 \\
\hline $\mathrm{C} 32-\mathrm{P} 2-\mathrm{Fe} 2$ & $112.5(2)$ & $\mathrm{C} 29-\mathrm{C} 28-\mathrm{C} 27$ & $119.8(7)$ \\
\hline $\mathrm{C} 5-\mathrm{S} 1-\mathrm{Fe} 2$ & $112.2(2)$ & $\mathrm{C} 29-\mathrm{C} 28-\mathrm{H} 28$ & 120.1 \\
\hline $\mathrm{C} 5-\mathrm{S} 1-\mathrm{Fe} 1$ & $116.5(2)$ & $\mathrm{C} 27-\mathrm{C} 28-\mathrm{H} 28$ & 120.1 \\
\hline
\end{tabular}




\begin{tabular}{|c|c|c|c|}
\hline $\mathrm{Fe} 2-\mathrm{S} 1-\mathrm{Fe} 1$ & $66.98(7)$ & $\mathrm{C} 28-\mathrm{C} 29-\mathrm{C} 30$ & $118.8(6)$ \\
\hline $\mathrm{C} 7-\mathrm{S} 2-\mathrm{Fe} 1$ & $111.5(2)$ & $\mathrm{C} 28-\mathrm{C} 29-\mathrm{H} 29$ & 120.6 \\
\hline $\mathrm{C} 7-\mathrm{S} 2-\mathrm{Fe} 2$ & $115.3(2)$ & $\mathrm{C} 30-\mathrm{C} 29-\mathrm{H} 29$ & 120.6 \\
\hline $\mathrm{Fe} 1-\mathrm{S} 2-\mathrm{Fe} 2$ & $67.31(7)$ & $\mathrm{C} 31-\mathrm{C} 30-\mathrm{C} 29$ & $121.2(6)$ \\
\hline $\mathrm{O} 1-\mathrm{C} 1-\mathrm{Fe} 1$ & $178.5(6)$ & $\mathrm{C} 31-\mathrm{C} 30-\mathrm{H} 30$ & 119.4 \\
\hline $\mathrm{O} 2-\mathrm{C} 2-\mathrm{Fe} 1$ & $175.9(6)$ & $\mathrm{C} 29-\mathrm{C} 30-\mathrm{H} 30$ & 119.4 \\
\hline $\mathrm{O} 3-\mathrm{C} 3-\mathrm{Fe} 2$ & $175.3(7)$ & $\mathrm{C} 30-\mathrm{C} 31-\mathrm{C} 26$ & $119.6(7)$ \\
\hline $\mathrm{O} 4-\mathrm{C} 4-\mathrm{Fe} 2$ & $179.0(7)$ & $\mathrm{C} 30-\mathrm{C} 31-\mathrm{H} 31$ & 120.2 \\
\hline $\mathrm{C} 6-\mathrm{C} 5-\mathrm{S} 1$ & $118.7(5)$ & $\mathrm{C} 26-\mathrm{C} 31-\mathrm{H} 31$ & 120.2 \\
\hline $\mathrm{C} 6-\mathrm{C} 5-\mathrm{H} 5 \mathrm{~A}$ & 107.6 & $\mathrm{C} 33-\mathrm{C} 32-\mathrm{C} 37$ & $119.7(7)$ \\
\hline $\mathrm{S} 1-\mathrm{C} 5-\mathrm{H} 5 \mathrm{~A}$ & 107.6 & $\mathrm{C} 33-\mathrm{C} 32-\mathrm{P} 2$ & $123.1(5)$ \\
\hline $\mathrm{C} 6-\mathrm{C} 5-\mathrm{H} 5 \mathrm{~B}$ & 107.6 & $\mathrm{C} 37-\mathrm{C} 32-\mathrm{P} 2$ & $117.0(6)$ \\
\hline $\mathrm{S} 1-\mathrm{C} 5-\mathrm{H} 5 \mathrm{~B}$ & 107.6 & $\mathrm{C} 32-\mathrm{C} 33-\mathrm{C} 34$ & $119.6(7)$ \\
\hline $\mathrm{H} 5 \mathrm{~A}-\mathrm{C} 5-\mathrm{H} 5 \mathrm{~B}$ & 107.1 & $\mathrm{C} 32-\mathrm{C} 33-\mathrm{H} 33$ & 120.2 \\
\hline $\mathrm{C} 7-\mathrm{C} 6-\mathrm{C} 5$ & $114.1(5)$ & $\mathrm{C} 34-\mathrm{C} 33-\mathrm{H} 33$ & 120.2 \\
\hline $\mathrm{C} 7-\mathrm{C} 6-\mathrm{H} 6 \mathrm{~A}$ & 108.7 & $\mathrm{C} 35-\mathrm{C} 34-\mathrm{C} 33$ & $119.1(7)$ \\
\hline $\mathrm{C} 5-\mathrm{C} 6-\mathrm{H} 6 \mathrm{~A}$ & 108.7 & $\mathrm{C} 35-\mathrm{C} 34-\mathrm{H} 34$ & 120.5 \\
\hline $\mathrm{C} 7-\mathrm{C} 6-\mathrm{H} 6 \mathrm{~B}$ & 108.7 & $\mathrm{C} 33-\mathrm{C} 34-\mathrm{H} 34$ & 120.5 \\
\hline $\mathrm{C} 5-\mathrm{C} 6-\mathrm{H} 6 \mathrm{~B}$ & 108.7 & $\mathrm{C} 36-\mathrm{C} 35-\mathrm{C} 34$ & $121.3(7)$ \\
\hline $\mathrm{H} 6 \mathrm{~A}-\mathrm{C} 6-\mathrm{H} 6 \mathrm{~B}$ & 107.6 & $\mathrm{C} 36-\mathrm{C} 35-\mathrm{H} 35$ & 119.4 \\
\hline $\mathrm{C} 6-\mathrm{C} 7-\mathrm{S} 2$ & $115.6(5)$ & $\mathrm{C} 34-\mathrm{C} 35-\mathrm{H} 35$ & 119.4 \\
\hline $\mathrm{C} 6-\mathrm{C} 7-\mathrm{H} 7 \mathrm{~A}$ & 108.4 & $\mathrm{C} 35-\mathrm{C} 36-\mathrm{C} 37$ & $120.3(7)$ \\
\hline $\mathrm{S} 2-\mathrm{C} 7-\mathrm{H} 7 \mathrm{~A}$ & 108.4 & $\mathrm{C} 35-\mathrm{C} 36-\mathrm{H} 36$ & 119.8 \\
\hline $\mathrm{C} 6-\mathrm{C} 7-\mathrm{H} 7 \mathrm{~B}$ & 108.4 & $\mathrm{C} 37-\mathrm{C} 36-\mathrm{H} 36$ & 119.8 \\
\hline $\mathrm{S} 2-\mathrm{C} 7-\mathrm{H} 7 \mathrm{~B}$ & 108.4 & $\mathrm{C} 36-\mathrm{C} 37-\mathrm{C} 32$ & $119.9(7)$ \\
\hline $\mathrm{H} 7 \mathrm{~A}-\mathrm{C} 7-\mathrm{H} 7 \mathrm{~B}$ & 107.4 & $\mathrm{C} 36-\mathrm{C} 37-\mathrm{H} 37$ & 120.0 \\
\hline $\mathrm{C} 9-\mathrm{C} 8-\mathrm{C} 13$ & $117.2(7)$ & $\mathrm{C} 32-\mathrm{C} 37-\mathrm{H} 37$ & 120.0 \\
\hline $\mathrm{C} 9-\mathrm{C} 8-\mathrm{P} 1$ & $122.0(5)$ & $\mathrm{C} 39-\mathrm{C} 38-\mathrm{C} 43$ & $117.0(7)$ \\
\hline $\mathrm{C} 13-\mathrm{C} 8-\mathrm{P} 1$ & $120.7(6)$ & $\mathrm{C} 39-\mathrm{C} 38-\mathrm{P} 2$ & $124.0(6)$ \\
\hline $\mathrm{C} 10-\mathrm{C} 9-\mathrm{C} 8$ & $123.4(7)$ & $\mathrm{C} 43-\mathrm{C} 38-\mathrm{P} 2$ & $118.6(6)$ \\
\hline $\mathrm{C} 10-\mathrm{C} 9-\mathrm{H} 9$ & 118.3 & $\mathrm{C} 38-\mathrm{C} 39-\mathrm{C} 40$ & $121.6(8)$ \\
\hline $\mathrm{C} 8-\mathrm{C} 9-\mathrm{H} 9$ & 118.3 & $\mathrm{C} 38-\mathrm{C} 39-\mathrm{H} 39$ & 119.2 \\
\hline $\mathrm{C} 9-\mathrm{C} 10-\mathrm{C} 11$ & $118.0(7)$ & $\mathrm{C} 40-\mathrm{C} 39-\mathrm{H} 39$ & 119.2 \\
\hline $\mathrm{C} 9-\mathrm{C} 10-\mathrm{H} 10$ & 121.0 & $\mathrm{C} 39-\mathrm{C} 40-\mathrm{C} 41$ & $119.5(8)$ \\
\hline $\mathrm{C} 11-\mathrm{C} 10-\mathrm{H} 10$ & 121.0 & $\mathrm{C} 39-\mathrm{C} 40-\mathrm{H} 40$ & 120.3 \\
\hline $\mathrm{C} 12-\mathrm{C} 11-\mathrm{C} 10$ & $119.6(7)$ & $\mathrm{C} 41-\mathrm{C} 40-\mathrm{H} 40$ & 120.3 \\
\hline $\mathrm{C} 12-\mathrm{C} 11-\mathrm{H} 11$ & 120.2 & $\mathrm{C} 42-\mathrm{C} 41-\mathrm{C} 40$ & $118.5(8)$ \\
\hline $\mathrm{C} 10-\mathrm{C} 11-\mathrm{H} 11$ & 120.2 & $\mathrm{C} 42-\mathrm{C} 41-\mathrm{H} 41$ & 120.8 \\
\hline $\mathrm{C} 11-\mathrm{C} 12-\mathrm{C} 13$ & $121.0(7)$ & $\mathrm{C} 40-\mathrm{C} 41-\mathrm{H} 41$ & 120.8 \\
\hline $\mathrm{C} 11-\mathrm{C} 12-\mathrm{H} 12$ & 119.5 & $\mathrm{C} 41-\mathrm{C} 42-\mathrm{C} 43$ & $122.6(8)$ \\
\hline $\mathrm{C} 13-\mathrm{C} 12-\mathrm{H} 12$ & 119.5 & $\mathrm{C} 41-\mathrm{C} 42-\mathrm{H} 42$ & 118.7 \\
\hline $\mathrm{C} 12-\mathrm{C} 13-\mathrm{C} 8$ & $120.9(8)$ & $\mathrm{C} 43-\mathrm{C} 42-\mathrm{H} 42$ & 118.7 \\
\hline $\mathrm{C} 12-\mathrm{C} 13-\mathrm{H} 13$ & 119.6 & $\mathrm{C} 42-\mathrm{C} 43-\mathrm{C} 38$ & $120.9(7)$ \\
\hline $\mathrm{C} 8-\mathrm{C} 13-\mathrm{H} 13$ & 119.6 & $\mathrm{C} 42-\mathrm{C} 43-\mathrm{H} 43$ & 119.6 \\
\hline $\mathrm{C} 19-\mathrm{C} 14-\mathrm{C} 15$ & $116.8(7)$ & $\mathrm{C} 38-\mathrm{C} 43-\mathrm{H} 43$ & 119.6 \\
\hline $\mathrm{C} 2-\mathrm{Fe} 1-\mathrm{Fe} 2-\mathrm{C} 4$ & $-0.7(3)$ & $\mathrm{Fe} 1-\mathrm{S} 1-\mathrm{C} 5-\mathrm{C} 6$ & $55.4(5)$ \\
\hline $\mathrm{C} 1-\mathrm{Fe} 1-\mathrm{Fe} 2-\mathrm{C} 4$ & $94.6(3)$ & $\mathrm{S} 1-\mathrm{C} 5-\mathrm{C} 6-\mathrm{C} 7$ & $-53.4(8)$ \\
\hline
\end{tabular}




\begin{tabular}{|c|c|c|c|}
\hline $\mathrm{P} 1-\mathrm{Fe} 1-\mathrm{Fe} 2-\mathrm{C} 4$ & $-133.1(3)$ & $\mathrm{C} 5-\mathrm{C} 6-\mathrm{C} 7-\mathrm{S} 2$ & $62.7(7)$ \\
\hline $\mathrm{S} 2-\mathrm{Fe} 1-\mathrm{Fe} 2-\mathrm{C} 4$ & $173.4(3)$ & $\mathrm{Fe} 1-\mathrm{S} 2-\mathrm{C} 7-\mathrm{C} 6$ & $-73.7(5)$ \\
\hline $\mathrm{S} 1-\mathrm{Fe} 1-\mathrm{Fe} 2-\mathrm{C} 4$ & $-80.4(3)$ & $\mathrm{Fe} 2-\mathrm{S} 2-\mathrm{C} 7-\mathrm{C} 6$ & $0.5(5)$ \\
\hline $\mathrm{C} 2-\mathrm{Fe} 1-\mathrm{Fe} 2-\mathrm{C} 3$ & $-92.5(4)$ & $\mathrm{C} 14-\mathrm{P} 1-\mathrm{C} 8-\mathrm{C} 9$ & $-112.5(7)$ \\
\hline $\mathrm{C} 1-\mathrm{Fe} 1-\mathrm{Fe} 2-\mathrm{C} 3$ & $2.8(3)$ & $\mathrm{C} 20-\mathrm{P} 1-\mathrm{C} 8-\mathrm{C} 9$ & $141.5(6)$ \\
\hline $\mathrm{P} 1-\mathrm{Fe} 1-\mathrm{Fe} 2-\mathrm{C} 3$ & $135.1(3)$ & $\mathrm{Fe} 1-\mathrm{P} 1-\mathrm{C} 8-\mathrm{C} 9$ & $15.8(7)$ \\
\hline $\mathrm{S} 2-\mathrm{Fe} 1-\mathrm{Fe} 2-\mathrm{C} 3$ & $81.6(3)$ & $\mathrm{C} 14-\mathrm{P} 1-\mathrm{C} 8-\mathrm{C} 13$ & $63.9(6)$ \\
\hline $\mathrm{S} 1-\mathrm{Fe} 1-\mathrm{Fe} 2-\mathrm{C} 3$ & $-172.2(3)$ & $\mathrm{C} 20-\mathrm{P} 1-\mathrm{C} 8-\mathrm{C} 13$ & $-42.1(6)$ \\
\hline $\mathrm{C} 2-\mathrm{Fe} 1-\mathrm{Fe} 2-\mathrm{P} 2$ & $126.3(3)$ & $\mathrm{Fe} 1-\mathrm{P} 1-\mathrm{C} 8-\mathrm{C} 13$ & $-167.8(5)$ \\
\hline $\mathrm{C} 1-\mathrm{Fe} 1-\mathrm{Fe} 2-\mathrm{P} 2$ & $-138.4(3)$ & $\mathrm{C} 13-\mathrm{C} 8-\mathrm{C} 9-\mathrm{C} 10$ & $-0.1(11)$ \\
\hline $\mathrm{P} 1-\mathrm{Fe} 1-\mathrm{Fe} 2-\mathrm{P} 2$ & $-6.0(3)$ & $\mathrm{P} 1-\mathrm{C} 8-\mathrm{C} 9-\mathrm{C} 10$ & $176.4(5)$ \\
\hline $\mathrm{S} 2-\mathrm{Fe} 1-\mathrm{Fe} 2-\mathrm{P} 2$ & $-59.59(19)$ & $\mathrm{C} 8-\mathrm{C} 9-\mathrm{C} 10-\mathrm{C} 11$ & $-1.4(11)$ \\
\hline $\mathrm{S} 1-\mathrm{Fe} 1-\mathrm{Fe} 2-\mathrm{P} 2$ & $46.63(18)$ & $\mathrm{C} 9-\mathrm{C} 10-\mathrm{C} 11-\mathrm{C} 12$ & $2.2(11)$ \\
\hline $\mathrm{C} 2-\mathrm{Fe} 1-\mathrm{Fe} 2-\mathrm{S} 1$ & $79.7(3)$ & $\mathrm{C} 10-\mathrm{C} 11-\mathrm{C} 12-\mathrm{C} 13$ & $-1.6(12)$ \\
\hline $\mathrm{C} 1-\mathrm{Fe} 1-\mathrm{Fe} 2-\mathrm{S} 1$ & $175.0(2)$ & $\mathrm{C} 11-\mathrm{C} 12-\mathrm{C} 13-\mathrm{C} 8$ & $0.1(12)$ \\
\hline $\mathrm{P} 1-\mathrm{Fe} 1-\mathrm{Fe} 2-\mathrm{S} 1$ & $-52.67(18)$ & $\mathrm{C} 9-\mathrm{C} 8-\mathrm{C} 13-\mathrm{C} 12$ & $0.7(11)$ \\
\hline $\mathrm{S} 2-\mathrm{Fe} 1-\mathrm{Fe} 2-\mathrm{S} 1$ & $-106.21(10)$ & $\mathrm{P} 1-\mathrm{C} 8-\mathrm{C} 13-\mathrm{C} 12$ & $-175.8(6)$ \\
\hline $\mathrm{C} 2-\mathrm{Fe} 1-\mathrm{Fe} 2-\mathrm{S} 2$ & $-174.1(3)$ & $\mathrm{C} 8-\mathrm{P} 1-\mathrm{C} 14-\mathrm{C} 19$ & $-72.6(7)$ \\
\hline $\mathrm{C} 1-\mathrm{Fe} 1-\mathrm{Fe} 2-\mathrm{S} 2$ & $-78.8(2)$ & $\mathrm{C} 20-\mathrm{P} 1-\mathrm{C} 14-\mathrm{C} 19$ & $32.5(7)$ \\
\hline $\mathrm{P} 1-\mathrm{Fe} 1-\mathrm{Fe} 2-\mathrm{S} 2$ & $53.54(18)$ & $\mathrm{Fe} 1-\mathrm{P} 1-\mathrm{C} 14-\mathrm{C} 19$ & $160.1(5)$ \\
\hline $\mathrm{S} 1-\mathrm{Fe} 1-\mathrm{Fe} 2-\mathrm{S} 2$ & $106.21(10)$ & $\mathrm{C} 8-\mathrm{P} 1-\mathrm{C} 14-\mathrm{C} 15$ & $101.6(6)$ \\
\hline $\mathrm{C} 2-\mathrm{Fe} 1-\mathrm{P} 1-\mathrm{C} 14$ & $159.2(4)$ & $\mathrm{C} 20-\mathrm{P} 1-\mathrm{C} 14-\mathrm{C} 15$ & $-153.3(5)$ \\
\hline $\mathrm{C} 1-\mathrm{Fe} 1-\mathrm{P} 1-\mathrm{C} 14$ & $65.3(4)$ & $\mathrm{Fe} 1-\mathrm{P} 1-\mathrm{C} 14-\mathrm{C} 15$ & $-25.7(7)$ \\
\hline $\mathrm{S} 2-\mathrm{Fe} 1-\mathrm{P} 1-\mathrm{C} 14$ & $-23.0(3)$ & $\mathrm{C} 19-\mathrm{C} 14-\mathrm{C} 15-\mathrm{C} 16$ & $0.6(11)$ \\
\hline $\mathrm{S} 1-\mathrm{Fe} 1-\mathrm{P} 1-\mathrm{C} 14$ & $-111.6(3)$ & $\mathrm{P} 1-\mathrm{C} 14-\mathrm{C} 15-\mathrm{C} 16$ & $-174.0(5)$ \\
\hline $\mathrm{Fe} 2-\mathrm{Fe} 1-\mathrm{P} 1-\mathrm{C} 14$ & $-67.8(3)$ & $\mathrm{C} 14-\mathrm{C} 15-\mathrm{C} 16-\mathrm{C} 17$ & $-3.0(11)$ \\
\hline $\mathrm{C} 2-\mathrm{Fe} 1-\mathrm{P} 1-\mathrm{C} 8$ & $39.7(4)$ & $\mathrm{C} 15-\mathrm{C} 16-\mathrm{C} 17-\mathrm{C} 18$ & $3.4(11)$ \\
\hline $\mathrm{C} 1-\mathrm{Fe} 1-\mathrm{P} 1-\mathrm{C} 8$ & $-54.2(3)$ & $\mathrm{C} 16-\mathrm{C} 17-\mathrm{C} 18-\mathrm{C} 19$ & $-1.4(12)$ \\
\hline $\mathrm{S} 2-\mathrm{Fe} 1-\mathrm{P} 1-\mathrm{C} 8$ & $-142.5(3)$ & $\mathrm{C} 17-\mathrm{C} 18-\mathrm{C} 19-\mathrm{C} 14$ & $-0.9(12)$ \\
\hline $\mathrm{S} 1-\mathrm{Fe} 1-\mathrm{P} 1-\mathrm{C} 8$ & $129.0(3)$ & $\mathrm{C} 15-\mathrm{C} 14-\mathrm{C} 19-\mathrm{C} 18$ & $1.3(11)$ \\
\hline $\mathrm{Fe} 2-\mathrm{Fe} 1-\mathrm{P} 1-\mathrm{C} 8$ & $172.8(3)$ & $\mathrm{P} 1-\mathrm{C} 14-\mathrm{C} 19-\mathrm{C} 18$ & $175.6(6)$ \\
\hline $\mathrm{C} 2-\mathrm{Fe} 1-\mathrm{P} 1-\mathrm{C} 20$ & $-79.1(3)$ & $\mathrm{C} 14-\mathrm{P} 1-\mathrm{C} 20-\mathrm{C} 25$ & $39.2(6)$ \\
\hline $\mathrm{C} 1-\mathrm{Fe} 1-\mathrm{P} 1-\mathrm{C} 20$ & $-173.1(3)$ & $\mathrm{C} 8-\mathrm{P} 1-\mathrm{C} 20-\mathrm{C} 25$ & $143.4(6)$ \\
\hline $\mathrm{S} 2-\mathrm{Fe} 1-\mathrm{P} 1-\mathrm{C} 20$ & $98.7(3)$ & $\mathrm{Fe} 1-\mathrm{P} 1-\mathrm{C} 20-\mathrm{C} 25$ & $-90.1(6)$ \\
\hline $\mathrm{S} 1-\mathrm{Fe} 1-\mathrm{P} 1-\mathrm{C} 20$ & $10.1(3)$ & $\mathrm{C} 14-\mathrm{P} 1-\mathrm{C} 20-\mathrm{C} 21$ & $-148.0(5)$ \\
\hline $\mathrm{Fe} 2-\mathrm{Fe} 1-\mathrm{P} 1-\mathrm{C} 20$ & $53.9(3)$ & $\mathrm{C} 8-\mathrm{P} 1-\mathrm{C} 20-\mathrm{C} 21$ & $-43.9(6)$ \\
\hline $\mathrm{C} 4-\mathrm{Fe} 2-\mathrm{P} 2-\mathrm{C} 26$ & $-154.4(4)$ & $\mathrm{Fe} 1-\mathrm{P} 1-\mathrm{C} 20-\mathrm{C} 21$ & $82.7(5)$ \\
\hline $\mathrm{C} 3-\mathrm{Fe} 2-\mathrm{P} 2-\mathrm{C} 26$ & $-63.6(4)$ & $\mathrm{C} 25-\mathrm{C} 20-\mathrm{C} 21-\mathrm{C} 22$ & $-1.3(10)$ \\
\hline $\mathrm{S} 1-\mathrm{Fe} 2-\mathrm{P} 2-\mathrm{C} 26$ & $116.5(3)$ & $\mathrm{P} 1-\mathrm{C} 20-\mathrm{C} 21-\mathrm{C} 22$ & $-174.4(5)$ \\
\hline $\mathrm{S} 2-\mathrm{Fe} 2-\mathrm{P} 2-\mathrm{C} 26$ & $27.7(3)$ & $\mathrm{C} 20-\mathrm{C} 21-\mathrm{C} 22-\mathrm{C} 23$ & $2.0(11)$ \\
\hline $\mathrm{Fe} 1-\mathrm{Fe} 2-\mathrm{P} 2-\mathrm{C} 26$ & $77.3(3)$ & $\mathrm{C} 21-\mathrm{C} 22-\mathrm{C} 23-\mathrm{C} 24$ & $-1.2(12)$ \\
\hline $\mathrm{C} 4-\mathrm{Fe} 2-\mathrm{P} 2-\mathrm{C} 38$ & $-35.2(3)$ & $\mathrm{C} 22-\mathrm{C} 23-\mathrm{C} 24-\mathrm{C} 25$ & $-0.2(11)$ \\
\hline $\mathrm{C} 3-\mathrm{Fe} 2-\mathrm{P} 2-\mathrm{C} 38$ & $55.6(4)$ & $\mathrm{C} 21-\mathrm{C} 20-\mathrm{C} 25-\mathrm{C} 24$ & $-0.1(10)$ \\
\hline $\mathrm{S} 1-\mathrm{Fe} 2-\mathrm{P} 2-\mathrm{C} 38$ & $-124.3(3)$ & $\mathrm{P} 1-\mathrm{C} 20-\mathrm{C} 25-\mathrm{C} 24$ & $172.7(5)$ \\
\hline $\mathrm{S} 2-\mathrm{Fe} 2-\mathrm{P} 2-\mathrm{C} 38$ & $146.9(3)$ & $\mathrm{C} 23-\mathrm{C} 24-\mathrm{C} 25-\mathrm{C} 20$ & $0.9(10)$ \\
\hline $\mathrm{Fe} 1-\mathrm{Fe} 2-\mathrm{P} 2-\mathrm{C} 38$ & $-163.6(3)$ & $\mathrm{C} 38-\mathrm{P} 2-\mathrm{C} 26-\mathrm{C} 27$ & $-129.7(6)$ \\
\hline $\mathrm{C} 4-\mathrm{Fe} 2-\mathrm{P} 2-\mathrm{C} 32$ & $82.3(3)$ & $\mathrm{C} 32-\mathrm{P} 2-\mathrm{C} 26-\mathrm{C} 27$ & $124.6(6)$ \\
\hline $\mathrm{C} 3-\mathrm{Fe} 2-\mathrm{P} 2-\mathrm{C} 32$ & $173.1(4)$ & $\mathrm{Fe} 2-\mathrm{P} 2-\mathrm{C} 26-\mathrm{C} 27$ & $-2.5(7)$ \\
\hline
\end{tabular}




\begin{tabular}{|c|c|c|c|}
\hline $\mathrm{S} 1-\mathrm{Fe} 2-\mathrm{P} 2-\mathrm{C} 32$ & $-6.8(3)$ & $\mathrm{C} 38-\mathrm{P} 2-\mathrm{C} 26-\mathrm{C} 31$ & $48.9(6)$ \\
\hline $\mathrm{S} 2-\mathrm{Fe} 2-\mathrm{P} 2-\mathrm{C} 32$ & $-95.5(3)$ & $\mathrm{C} 32-\mathrm{P} 2-\mathrm{C} 26-\mathrm{C} 31$ & $-56.8(6)$ \\
\hline $\mathrm{Fe} 1-\mathrm{Fe} 2-\mathrm{P} 2-\mathrm{C} 32$ & $-46.0(3)$ & $\mathrm{Fe} 2-\mathrm{P} 2-\mathrm{C} 26-\mathrm{C} 31$ & $176.1(5)$ \\
\hline $\mathrm{C} 4-\mathrm{Fe} 2-\mathrm{S} 1-\mathrm{C} 5$ & $-145.0(3)$ & $\mathrm{C} 31-\mathrm{C} 26-\mathrm{C} 27-\mathrm{C} 28$ & $1.1(11)$ \\
\hline $\mathrm{C} 3-\mathrm{Fe} 2-\mathrm{S} 1-\mathrm{C} 5$ & $128.1(6)$ & $\mathrm{P} 2-\mathrm{C} 26-\mathrm{C} 27-\mathrm{C} 28$ & $179.7(5)$ \\
\hline $\mathrm{P} 2-\mathrm{Fe} 2-\mathrm{S} 1-\mathrm{C} 5$ & $-52.1(3)$ & $\mathrm{C} 26-\mathrm{C} 27-\mathrm{C} 28-\mathrm{C} 29$ & $1.8(11)$ \\
\hline $\mathrm{S} 2-\mathrm{Fe} 2-\mathrm{S} 1-\mathrm{C} 5$ & $57.5(3)$ & $\mathrm{C} 27-\mathrm{C} 28-\mathrm{C} 29-\mathrm{C} 30$ & $-3.4(11)$ \\
\hline $\mathrm{Fe} 1-\mathrm{Fe} 2-\mathrm{S} 1-\mathrm{C} 5$ & $110.5(3)$ & $\mathrm{C} 28-\mathrm{C} 29-\mathrm{C} 30-\mathrm{C} 31$ & $2.2(11)$ \\
\hline $\mathrm{C} 4-\mathrm{Fe} 2-\mathrm{S} 1-\mathrm{Fe} 1$ & $104.5(2)$ & $\mathrm{C} 29-\mathrm{C} 30-\mathrm{C} 31-\mathrm{C} 26$ & $0.6(11)$ \\
\hline $\mathrm{C} 3-\mathrm{Fe} 2-\mathrm{S} 1-\mathrm{Fe} 1$ & $17.6(6)$ & $\mathrm{C} 27-\mathrm{C} 26-\mathrm{C} 31-\mathrm{C} 30$ & $-2.2(10)$ \\
\hline $\mathrm{P} 2-\mathrm{Fe} 2-\mathrm{S} 1-\mathrm{Fe} 1$ & $-162.59(8)$ & $\mathrm{P} 2-\mathrm{C} 26-\mathrm{C} 31-\mathrm{C} 30$ & $179.2(5)$ \\
\hline $\mathrm{S} 2-\mathrm{Fe} 2-\mathrm{S} 1-\mathrm{Fe} 1$ & $-53.01(7)$ & $\mathrm{C} 26-\mathrm{P} 2-\mathrm{C} 32-\mathrm{C} 33$ & $-11.0(6)$ \\
\hline $\mathrm{C} 2-\mathrm{Fe} 1-\mathrm{S} 1-\mathrm{C} 5$ & $151.2(3)$ & $\mathrm{C} 38-\mathrm{P} 2-\mathrm{C} 32-\mathrm{C} 33$ & $-114.3(5)$ \\
\hline $\mathrm{C} 1-\mathrm{Fe} 1-\mathrm{S} 1-\mathrm{C} 5$ & $-116.7(6)$ & $\mathrm{Fe} 2-\mathrm{P} 2-\mathrm{C} 32-\mathrm{C} 33$ & $120.5(5)$ \\
\hline $\mathrm{P} 1-\mathrm{Fe} 1-\mathrm{S} 1-\mathrm{C} 5$ & $55.4(2)$ & $\mathrm{C} 26-\mathrm{P} 2-\mathrm{C} 32-\mathrm{C} 37$ & $173.8(5)$ \\
\hline $\mathrm{S} 2-\mathrm{Fe} 1-\mathrm{S} 1-\mathrm{C} 5$ & $-50.2(2)$ & $\mathrm{C} 38-\mathrm{P} 2-\mathrm{C} 32-\mathrm{C} 37$ & $70.6(5)$ \\
\hline $\mathrm{Fe} 2-\mathrm{Fe} 1-\mathrm{S} 1-\mathrm{C} 5$ & $-104.3(2)$ & $\mathrm{Fe} 2-\mathrm{P} 2-\mathrm{C} 32-\mathrm{C} 37$ & $-54.6(5)$ \\
\hline $\mathrm{C} 2-\mathrm{Fe} 1-\mathrm{S} 1-\mathrm{Fe} 2$ & $-104.5(2)$ & $\mathrm{C} 37-\mathrm{C} 32-\mathrm{C} 33-\mathrm{C} 34$ & $-0.7(9)$ \\
\hline $\mathrm{C} 1-\mathrm{Fe} 1-\mathrm{S} 1-\mathrm{Fe} 2$ & $-12.4(6)$ & $\mathrm{P} 2-\mathrm{C} 32-\mathrm{C} 33-\mathrm{C} 34$ & $-175.7(4)$ \\
\hline $\mathrm{P} 1-\mathrm{Fe} 1-\mathrm{S} 1-\mathrm{Fe} 2$ & $159.70(8)$ & $\mathrm{C} 32-\mathrm{C} 33-\mathrm{C} 34-\mathrm{C} 35$ & $2.3(9)$ \\
\hline $\mathrm{S} 2-\mathrm{Fe} 1-\mathrm{S} 1-\mathrm{Fe} 2$ & $54.07(7)$ & $\mathrm{C} 33-\mathrm{C} 34-\mathrm{C} 35-\mathrm{C} 36$ & $-2.5(10)$ \\
\hline $\mathrm{C} 2-\mathrm{Fe} 1-\mathrm{S} 2-\mathrm{C} 7$ & $124.4(7)$ & $\mathrm{C} 34-\mathrm{C} 35-\mathrm{C} 36-\mathrm{C} 37$ & $1.0(11)$ \\
\hline $\mathrm{C} 1-\mathrm{Fe} 1-\mathrm{S} 2-\mathrm{C} 7$ & $-145.6(3)$ & $\mathrm{C} 35-\mathrm{C} 36-\mathrm{C} 37-\mathrm{C} 32$ & $0.7(10)$ \\
\hline $\mathrm{P} 1-\mathrm{Fe} 1-\mathrm{S} 2-\mathrm{C} 7$ & $-50.0(3)$ & $\mathrm{C} 33-\mathrm{C} 32-\mathrm{C} 37-\mathrm{C} 36$ & $-0.8(9)$ \\
\hline $\mathrm{S} 1-\mathrm{Fe} 1-\mathrm{S} 2-\mathrm{C} 7$ & $56.0(3)$ & $\mathrm{P} 2-\mathrm{C} 32-\mathrm{C} 37-\mathrm{C} 36$ & $174.5(5)$ \\
\hline $\mathrm{Fe} 2-\mathrm{Fe} 1-\mathrm{S} 2-\mathrm{C} 7$ & $109.5(3)$ & $\mathrm{C} 26-\mathrm{P} 2-\mathrm{C} 38-\mathrm{C} 39$ & $-96.6(6)$ \\
\hline $\mathrm{C} 2-\mathrm{Fe} 1-\mathrm{S} 2-\mathrm{Fe} 2$ & $14.9(6)$ & $\mathrm{C} 32-\mathrm{P} 2-\mathrm{C} 38-\mathrm{C} 39$ & $10.7(6)$ \\
\hline $\mathrm{C} 1-\mathrm{Fe} 1-\mathrm{S} 2-\mathrm{Fe} 2$ & $104.9(2)$ & $\mathrm{Fe} 2-\mathrm{P} 2-\mathrm{C} 38-\mathrm{C} 39$ & $133.6(5)$ \\
\hline $\mathrm{P} 1-\mathrm{Fe} 1-\mathrm{S} 2-\mathrm{Fe} 2$ & $-159.49(8)$ & $\mathrm{C} 26-\mathrm{P} 2-\mathrm{C} 38-\mathrm{C} 43$ & $76.7(6)$ \\
\hline $\mathrm{S} 1-\mathrm{Fe} 1-\mathrm{S} 2-\mathrm{Fe} 2$ & $-53.49(7)$ & $\mathrm{C} 32-\mathrm{P} 2-\mathrm{C} 38-\mathrm{C} 43$ & $-176.1(5)$ \\
\hline $\mathrm{C} 4-\mathrm{Fe} 2-\mathrm{S} 2-\mathrm{C} 7$ & $-120.2(6)$ & $\mathrm{Fe} 2-\mathrm{P} 2-\mathrm{C} 38-\mathrm{C} 43$ & $-53.2(6)$ \\
\hline $\mathrm{C} 3-\mathrm{Fe} 2-\mathrm{S} 2-\mathrm{C} 7$ & $154.6(3)$ & $\mathrm{C} 43-\mathrm{C} 38-\mathrm{C} 39-\mathrm{C} 40$ & $2.5(10)$ \\
\hline $\mathrm{P} 2-\mathrm{Fe} 2-\mathrm{S} 2-\mathrm{C} 7$ & $54.6(2)$ & $\mathrm{P} 2-\mathrm{C} 38-\mathrm{C} 39-\mathrm{C} 40$ & $175.8(5)$ \\
\hline $\mathrm{S} 1-\mathrm{Fe} 2-\mathrm{S} 2-\mathrm{C} 7$ & $-50.1(2)$ & $\mathrm{C} 38-\mathrm{C} 39-\mathrm{C} 40-\mathrm{C} 41$ & $-3.2(11)$ \\
\hline $\mathrm{Fe} 1-\mathrm{Fe} 2-\mathrm{S} 2-\mathrm{C} 7$ & $-104.0(2)$ & $\mathrm{C} 39-\mathrm{C} 40-\mathrm{C} 41-\mathrm{C} 42$ & $2.3(11)$ \\
\hline $\mathrm{C} 4-\mathrm{Fe} 2-\mathrm{S} 2-\mathrm{Fe} 1$ & $-16.2(6)$ & $\mathrm{C} 40-\mathrm{C} 41-\mathrm{C} 42-\mathrm{C} 43$ & $-0.9(12)$ \\
\hline $\mathrm{C} 3-\mathrm{Fe} 2-\mathrm{S} 2-\mathrm{Fe} 1$ & $-101.4(2)$ & $\mathrm{C} 41-\mathrm{C} 42-\mathrm{C} 43-\mathrm{C} 38$ & $0.2(11)$ \\
\hline $\mathrm{P} 2-\mathrm{Fe} 2-\mathrm{S} 2-\mathrm{Fe} 1$ & $158.63(8)$ & $\mathrm{C} 39-\mathrm{C} 38-\mathrm{C} 43-\mathrm{C} 42$ & $-1.0(10)$ \\
\hline $\mathrm{S} 1-\mathrm{Fe} 2-\mathrm{S} 2-\mathrm{Fe} 1$ & $53.90(7)$ & $\mathrm{P} 2-\mathrm{C} 38-\mathrm{C} 43-\mathrm{C} 42$ & $-174.7(5)$ \\
\hline $\mathrm{Fe} 2-\mathrm{S} 1-\mathrm{C} 5-\mathrm{C} 6$ & $-19.0(6)$ & & \\
\hline
\end{tabular}

